\title{
Somatic Symptoms in Depression and Their Correlation with Suicidality - A Cross-Sectional Study
}

\author{
Tehmina Asif ${ }^{1}$, Druhin Adavalath², Padmini Cherukunnath ${ }^{3}$ \\ 1Department of Psychiatry, Government Medical College, Kannur, Kerala, India. \\ 2Department of Psychiatry, Government Medical College, Kannur, Kerala, India. \\ ${ }^{3}$ Department of Psychiatry, Government Medical College, Kannur, Kerala, India.
}

\section{ABSTRACT}

\section{BACKGROUND}

Diagnostic criteria for MDD is based on psychological and vegetative symptoms. However, other somatic and pain symptoms are frequent in these patients. We wanted to study the prevalence of somatic symptoms in $1^{\text {st }}$ episode of depression and assess its correlation with suicidality.

\section{METHODS}

Patients aged 18 - 65 yrs. attending psychiatry outpatient department and admitted in wards, meeting the inclusion criteria were included in the study. A total of 177 patients was included. Patients were assessed with sociodemographic proforma. M.I.N.I. was used to diagnose major depressive disorder and to rule out the presence of other comorbid psychiatric illness. PHQ - 15 was used to detect the presence of somatic symptoms. Suicidality was assessed with Beck's Hopelessness Scale. Statistical analysis was done using SPSS. Descriptive statistics, Kruskal Wallis Test, Fisher's Exact Test, Chi - Squared Test, and Spearman correlation.

\section{RESULTS}

There is $100 \%$ prevalence of somatic symptoms in depression out of which Somatic Symptom Severity was minimal in $11.9 \%$, low in $54.2 \%$, medium in $32.2 \%$, and severe in $1.7 \%$. There was a strong positive correlation between PHQ - 15 Score and BHS Score, and this correlation was statistically significant ( $r$ o $=0.61, \mathrm{p}=<0.001$ ).

\section{CONCLUSIONS}

Many patients presenting predominantly with somatic complaints are misdiagnosed when they are evaluated in the primary care or medical clinics. In view of the high prevalence of somatic symptoms in depression, it is important to include these symptoms in the diagnostic criteria of depression, so as to increase the sensitivity and specificity of the diagnosis.

\section{KEY WORDS}

Depression, Somatic Symptoms, Suicidality
Corresponding Author: Dr. Druhin Adavalath, Professor, Government Medical College, Kannur, Kerala, India. E-mail:druhinav@yahoo.co.in

DOI: $10.14260 / \mathrm{jemds} / 2020 / 615$

How to Cite This Article:

Asif T, Adavalath D. Cherukunnath $P$. Somatic symptoms in depression and their correlation with suicidality - a cross sectional study. J Evolution Med Dent Sci 2020;9(38): 2824-2828, DOI: $10.14260 /$ jemds/2020/615

Submission 20-05-2020,

Peer Review 14-08-2020,

Acceptance 22-08-2020,

Published 21-09-2020.

Copyright (C) 2020 Tehmina Asif et al. This is an open access article distributed under Creative Commons Attribution License [Attribution 4.0 International (CC BY 4.0)] 


\section{BACKGROUND}

Major depression is a serious, recurrent disorder linked to diminished role functioning and quality of life, medical morbidity, and mortality. The World Health Organization ranks depression as the fourth leading cause of disability worldwide, ${ }^{1}$ and projects that by 2020 , it will be the second leading cause. Recently conducted world mental health surveys indicate that major depression is experienced by 10 $15 \%$ people in their lifetime ${ }^{2}$ and about $5 \%$ suffer from major depression in any given year. Lifetime prevalence of all depressive disorders taken together is more than $20 \%$, which is one in five individuals. ${ }^{3}$ In Indian context, a recent large sample survey conducted reported an overall prevalence of $15.9 \%$ for depression, ${ }^{4}$ which is similar to western figures.

There is some suggestion that perhaps the prevalence of depression has increased over past few decades. Studies done in primary health care settings in India have found depression in $21-84 \%$ of the cases.5,6 Though there are various typical signs and symptoms in depression, it may commonly disguise in somatic sign and symptoms and this is the reason for diagnosis to become difficult, wrong diagnosis, unnecessary and costly investigations and poor treatment outcome. These patients experience and describe emotional distress in terms of physical symptoms. A high percentage of patients with depression who seek treatment in a primary care setting report mainly physical symptoms, which can make depression very difficult to diagnose.7,8

The presence of pain predicts a longer time to remission and the resolution of these physical symptoms is important as it is strong predictor of full remission. Failure to address the physical symptoms associated with depression may compromise and reduce the overall remission rate. Also, patients who achieve remission but continue to suffer from residual physical symptoms may have a greater risk of clinical relapse of depression. ${ }^{9,10,11,12}$ Suicide is a major preventable cause of death worldwide. The first ever mental health action plan by WHO which was created in May 2013 has suicide prevention as an integral part of the plan. The aim is to reduce the rate of suicides in countries by $10 \%$ by $2020 .{ }^{13} \mathrm{~A}$ previous suicide attempt is considered as an important risk factor for further suicide attempts. Similarly, hopelessness and intentionality of suicide are considered as important predictors of future suicide attempts. ${ }^{14}$ So early identification of cause for suicide attempts and level of suicidal intent play an important role in successful creation of suicide prevention strategies.

\section{Objectives}

1. To estimate the prevalence of somatic symptoms in $1^{\text {st }}$ episode of depression

2. To assess the correlation between somatic symptoms and suicidality

\section{METHODS}

This is a hospital based cross sectional study conducted over a period of 1 year from 2018 March to 2019 February.

\section{Study Population}

Consecutive patients in the age group of 18 - 65 years diagnosed to have $1^{\text {st }}$ episode depression in Inpatient and outpatient department of psychiatry and also patients admitted in other wards consulting psychiatry department of Pariyaram Medical College.

\section{Study Sample}

A consecutive series of 177 patients who satisfied the inclusion and exclusion criteria were recruited in the study. Sample size was calculated using the formula $4 \mathrm{pq} / \mathrm{d}^{2}$ with an assured prevalence of $77 \%$ for somatic complaints and a precision factor of five.

\section{Inclusion Criteria}

- Patients of either gender of age group $18-65$ yrs. who is diagnosed with 1st major depressive episode by mini international neuropsychiatric interview.

- $\quad$ Those patients who are willing to give informed written consent.

\section{Exclusion Criteria}

- Severe depression with psychotic symptoms.

- $\quad$ Other co - morbid psychiatric illness.

- Chronic debilitating physical illness - diabetes, hypertension, cerebrovascular disease.

- Physical illness which could explain the reported somatic complaints.

- Dementia.

\section{Assessment Tools and Instruments}

- Socio - demographic data sheet.

- MINI international neuropsychiatry interview.

- $\quad$ Patient Health Questionnaire - 15.

- Hamilton depression scale.

- Beck's hopelessness scale.

\section{Data Collection Method}

After getting clearance from the institutional ethical committee, all consecutive patients aged 18 - 65 yrs. from inpatient and outpatient department of psychiatry and also patient admitted in the other wards consulting psychiatry department of GMC, Kannur during the period from March 2018 to February 2019 who satisfy the inclusion criteria was approached for the study. The need for the study, its aims and methods were explained to the patients. Those patients giving a written informed consent and satisfying the inclusion and exclusion criteria were assessed with sociodemographic Performa specially prepared by the researcher for this study. M.I.N.I. was used to diagnose major depressive disorder and to rule out the presence of other comorbid psychiatric illness. PHQ - 15 was used to detect the presence of somatic symptoms. Severity of depressive disorder was assessed with HAM - D. Suicidality assessed with Beck's Hopelessness Scale. ${ }^{15,16}$ 


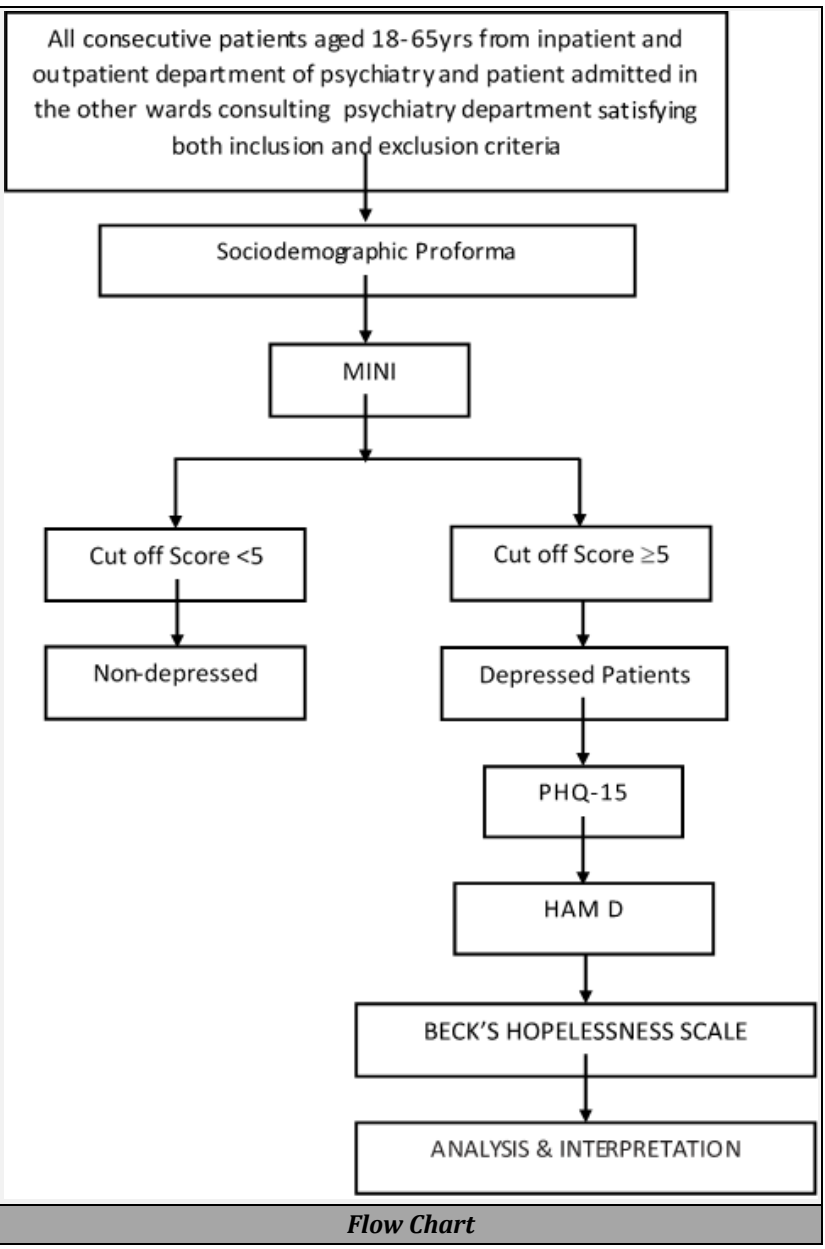

\section{Statistical Analysis}

Descriptive statistic tools were used to assess mean, median, standard deviation, percentage, interquartile range. Descriptive statistics, Kruskal Wallis Test, Fisher's Exact Test, Chi - Squared Test, Spearman correlation was used. Continuous variables was analyzed using student's t test. P value $<0.05$ will be considered clinically significant. Spearman's rank correlation coefficient was used to study the correlation of somatic symptoms with severity of depression and suicidal ideation. Data analysis was done using SPSS software.

\section{RESULTS}

\section{Distribution of the Participants in Terms of Age (Years)}

The mean (SD) of age (years) was 37.36 (10.73). The median (IQR) of age (years) was 36.00 (15.00). The age (years) ranged from 19 - 59.

\begin{tabular}{|ccc|}
\hline Somatic Symptom Severity & Frequency & Percentage \\
Minimal & 21 & $11.9 \%$ \\
Low & 96 & $54.2 \%$ \\
Medium & 57 & $32.2 \%$ \\
Severe & 3 & $1.7 \%$ \\
Total & $\mathbf{1 7 7}$ & $\mathbf{1 0 0 . 0} \%$ \\
\hline Table 1. Distribution of the Participants in Terms of \\
Somatic Symptom Severity (n = 177) \\
\hline
\end{tabular}
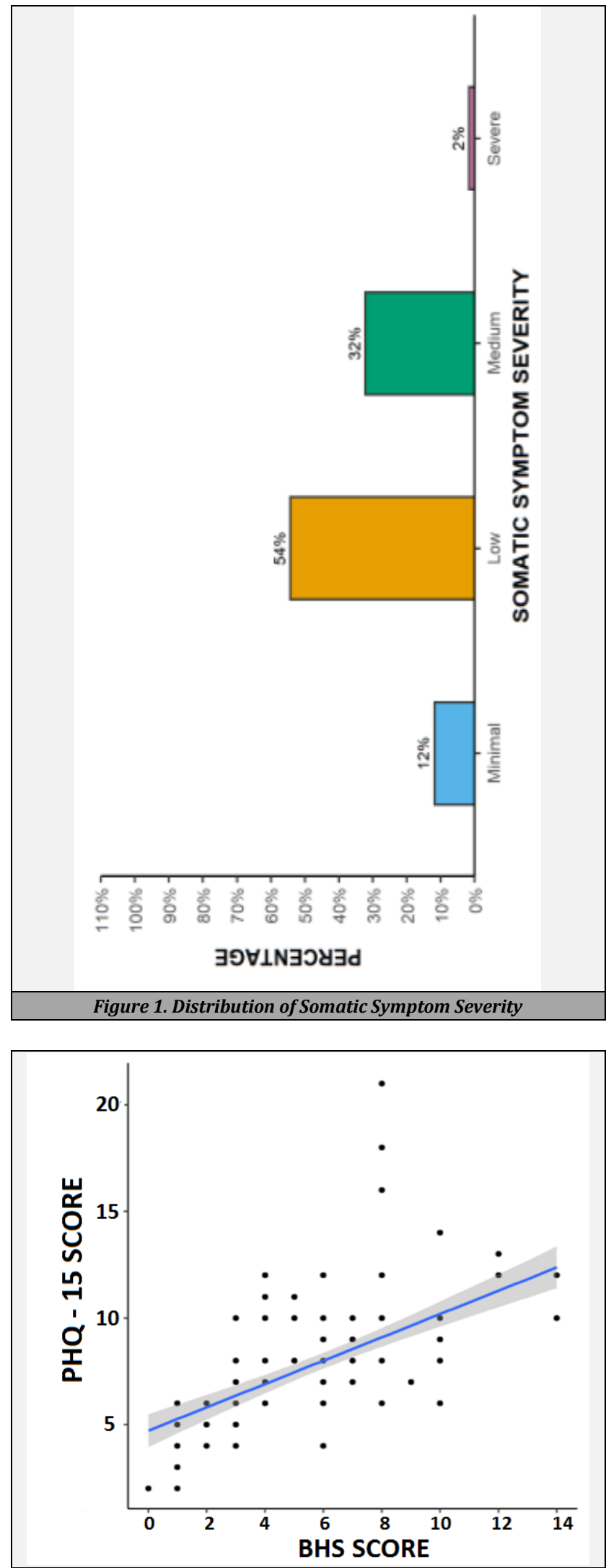

Figure 2. Correlation between BHS Score and PHQ - 15 Score $(n=177)$

Distribution of the Participants in Terms of Gender

The gender distribution showed that majority was female i.e. $39.0 \%$ Male and $61.0 \%$ Female. $11.9 \%$ of the participants had Somatic Symptom Severity: Minimal. 54.2 \% of the participants had Somatic Symptom Severity: Low. $32.2 \%$ of the participants had Somatic Symptom Severity: Medium. 1.7 
$\%$ of the participants had Somatic Symptom Severity: Severe. $25.4 \%$ of the participants had Hopelessness: None/Minimal. $52.5 \%$ of the participants had Hopelessness: Mild. $22.0 \%$ of the participants had Hopelessness: Moderate.

The above scatterplot depicts the correlation between BHS Score and PHQ - 15 Score. Individual points represent individual cases. The blue trendline represents the general trend of correlation between the two variables. The shaded grey area represents the $95 \%$ confidence interval of this trendline. Non - parametric tests (Spearman Correlation) were used to explore the correlation between the two variables, as at least one of the variables was not normally distributed. There was a strong positive correlation between BHS Score and PHQ - 15 Score, and this correlation was statistically significant ( $r h o=0.61, p=<0.001$ ). For every 1 unit increase in BHS Score, the PHQ - 15 Score increases by 0.55 units.

\section{DISCUSSION}

A hospital based cross - sectional study was done on patients diagnosed with $1^{\text {st }}$ episode depression who satisfied our inclusion and exclusion criteria.

\section{Sociodemographic Profile}

Our study included 177 patients of age $18-65$ yrs. diagnosed with $1^{\text {st }}$ episode of depression. In 177 patients, 69 (39.0\%) were males and 108 (61.0 \%) were females. Females reported higher somatic symptoms. This finding is similar to many international (Silverstien et al 1999, Belbase et al 2014, Zimmerman et al 2018.17,18 and Indian studies (Amin et al 1998, Poongothai et al 2009).4,6 But in IPS multicentre study there was equal gender distribution. ${ }^{19}$ The mean (SD) of Age (Years) was 37.36 (10.73). It was found there was positive correlation between age and somatic symptoms. Supported by some studies, like the study by Ohayon, Maurice et al 2010; Mutsuhirom, Nakao et al 2005.20,21

\section{Prevalence of Somatic Symptoms}

In this study, we have used 15 - item patient health questionnaire physical symptoms (PHQ - 15) to assess somatic symptoms. There was $100 \%$ prevalence of somatic symptoms in depression and out of which Somatic Symptom Severity: 11.9 \% - Minimal. $54.2 \%$ - Low. $32.2 \%$ - Medium. $1.7 \%$ Severe. The most common somatic symptom being low energy (98.8\%) closely followed by trouble sleeping (90.4\%), $68.3 \%$ had pain of arms, legs, joints, headaches (45.2\%). In the latest study in Asia (2018), major somatic symptoms in patients with MDD are insomnia, pre - verbal physical complaints, weight loss, low appetite, circulatory system complaints, headache, hyposexuality, gastrointestinal system complaints, and respiratory system complaints. These symptoms vary by the type of medical setting to which patients present, and well as by age, and gender. ${ }^{22}$

In one of the recent studies from India, the most commonly reported FSCs were lack of energy (weakness) much of the time (76.2\%), severe headache (74\%), and feeling tired when not working $(71 \%)$. Other commonly reported symptoms present in about half of the sample were pain in legs (64\%), aware of palpitations (59.5\%), head feeling heavy (59.4\%), aches and pains all over the body (55.5\%), mouth or throat getting dry (55.2\%), pain or tension in neck and shoulder (54 $\%$ ), head feeling hot or burning (54\%), and darkness or mist in front of the eyes (49.1\%). ${ }^{19}$

\section{Correlation of Somatic Symptoms with Suicidality}

A positive correlation was found between suicidality and somatic symptom. There was a strong positive correlation between PHQ - 15 Score and BHS Score, and this correlation was statistically significant ( $\mathrm{rho}=0.61, \mathrm{p}=<0.001$ ). This was supported by a recent study Zimmerman et al in 2018, Bahk, Won Myong Park et al 2011. However, the STRA*D study (Husain et al., 2007) for patients with MDD who have not received a medical diagnosis of pain conditions found that suicidal ideation and suicide attempts were not associated with pain. There are not much studies from India, assessing the correlation between suicidality and somatic symptoms.

\section{CONCLUSIONS}

Females reported higher somatic symptoms. (61 \%). The prevalence of somatic symptoms was found to be $100 \%$. Somatic symptom severity predominantly ranged from low $(54.2 \%)$ to medium (32.2\%). There is a positive correlation between severity of depression and suicidality.

Financial or Other Competing Interests: None.

\section{REFERENCES}

[1] Michaud CM, Murray CJ, Bloom BR. Burden of disease-implications for future research. JAMA 2001;285(5):5359.

[2] Bromet E, Andrade LH, Hwang I, et al. Cross-national epidemiology of DSM - IV major depressive episode. BMC Med 2011;9:90.

[3] Murphy JM, Laird NM, Monson RR, et al. A 40 - year perspective on the prevalence of depression: the stirling county study. Arch Gen Psychiatry 2000;57(3):209-15.

[4] Poongothai S, Pradeepa R, Ganesan A, et al. Prevalence of depression in a large urban south Indian population -- the Chennai urban rural epidemiology study (CURES - 70). PLoS One 2009;4(9):e7185.

[5] Nandi DN, Banerjee G, Mukherjee SP, et al. Psychiatric morbidity of a rural Indian community. Changes over a 20 - year interval. Br J Psychiatry 2000;176:351-6.

[6] Amin G, Shah S, Vankar GK. The prevelance and recognition of depression in primary care. Indian J Psychiatry 1998;40(4):364-9.

[7] Novick D, Montgomery W, Aguado J, et al. Which somatic symptoms are associated with an unfavorable course in Asian patients with major depressive disorder? J Affect Disord 2013;149(1-3):182-8.

[8] Sayar K, Kirmayer LJ, Taillefer SS. Predictors of somatic symptoms in depressive disorder. Gen Hosp Psychiatry 2003;25(2):108-14. 
[9] Paykel ES. Partial remission, residual symptoms and relapse in depression. Dialogues Clin Neurosci 2008;10(4):431-7.

[10] Greco T, Eckert G, Kroenke K. The outcome of physical symptoms with treatment of depression. J Gen Intern Med 2004;19(8):813-8.

[11] Bair MJ, Robinson RL, Katon W, et al. Depression and pain comorbidity: a literature review. Arch Intern Med 2003;163(20):2433-45.

[12] Paykel ES, Scott J, Teasdale JD, et al. Prevention of relapse in residual depression by cognitive therapy: a controlled trial. Arch Gen Psychiatry 1999;56(9):829-35.

[13] World Health Organization. Preventing suicide: a global imperative executive summary. Geneva: WHO Press 2014.

[14] Menon V. Suicide risk assessment and formulation : an update. Assian J Psychaitr 2013;6(5):430-5.

[15] Hamilton M. Frequency of symptoms in melancholia (Depressive Illness). Br J Psychiatry 1989;154:201-6.

[16] Kroenke K, Spitzer RL, Williams JBW. The PHQ - 15: validity of a new measure for evaluating the severity of somatic symptoms. Psychosom Med 2002;(64):258-66.
[17] Zimmerman M, Balling C, Chelminski I, et al. Understanding the severity of depression: which symptoms of depression are the best indicators of depression severity? Compr Psychiatry 2018;87:84-8.

[18] Silverstein B. Gender difference in the prevalence of clinical depression: the role played by depression associated with somatic symptoms. Am J Psychiatry 1999;156(3):480-2.

[19] Grover S, Avasthi A, Kalita K, et al. IPS multicentric study: functional somatic symptoms in depression. Indian J Psychiatry 2013;55(1):31-40.

[20] Ohayon MM, Schatzberg AF. Chronic pain and major depressive disorder in the general population. J Psychiatr Res 2010;44(7):454-61.

[21] Nakao M, Yano E. Somatic symptoms for predicting depression: one - year follow - up study in annual health examinations. Psychiatry Clin Neurosci 2006;60(2):21925.

[22] Srinivasan K, Murthy RS, Janakiramaiah N. A nosological study of patients presenting with somatic complaints. Acta Psychiatr Scand 1986;73(1):1-5. 